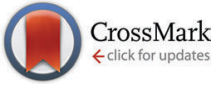

Cite this: Chem. Commun., 2015, 51, 11899

Received 11th May 2015,

Accepted 16th June 2015

DOI: $10.1039 / \mathrm{c5cc03901k}$

www.rsc.org/chemcomm

\section{Renaissance for low shrinking resins: all-in-one solution by bi-functional vinylcyclopropane-amides $\uparrow$}

\author{
Paul Pineda Contreras, ${ }^{a}$ Christian Kuttner, ${ }^{\mathrm{b}}$ Andreas Fery, ${ }^{\mathrm{b}}$ Ullrich Stahlschmidt, ${ }^{\mathrm{c}}$ \\ Valérie Jérôme, ${ }^{c}$ Ruth Freitag ${ }^{c}$ and Seema Agarwal*a
}

\begin{abstract}
A low volume shrinking vinylcyclopropane (VCP) monomer, showing both a high reactivity and a low viscosity, was obtained by applying a sterically hindered and isomeric spacer element, incorporating intermolecular amide hydrogen bonds. The resulting properties locate this VCP system in a pronounced range that so far no other efficient and radical polymerizable resin could enter.
\end{abstract}

Photo-polymerizable resins with a low volume shrinkage are fundamental for coatings, ${ }^{1}$ electronics, ${ }^{2}$ microlithography, ${ }^{3}$ holographic data storages, ${ }^{4}$ advanced composites, ${ }^{5}$ and dental applications. ${ }^{6}$ In these applications, the final properties of the cross-linked network have to match precise specifications adapted for the final end-use. In general, this precision is achieved by the selection of highly specified resin systems. ${ }^{7}$ Thereby, in many cases the fast and selective curing, the solvent-free formulation and the targeted control of curing over a wide temperature, time, and radiation range made in particular the photo-polymerization technique overwhelming. ${ }^{8}$ Thus, especially acrylate and methacrylate monomers are favoured in nearly all radically polymerizable resins due to their synthetic ease of access, processability, and chemical variability. ${ }^{9}$

However, the observed volume shrinkage is pronounced and inevitable due to the transition of the intermolecular interactions from van der Waals spheres for monomers to covalent bonds in the cured resins. ${ }^{10}$ As a result, a high shrinkage can lead to the reduction in adhesion between the coating layer and the substrate in coating applications, or may cause the formation of contraction gaps between filling and cavity in dental resins, whereby recurrent caries might be the consequence. ${ }^{11}$

\footnotetext{
${ }^{a}$ Macromolecular Chemistry II and Bayreuth Center for Colloids and Interfaces, Universität Bayreuth, Universitätsstrasse 30, 95440 Bayreuth, Germany.

E-mail: agarwal@uni-bayreuth.de; Tel: +49-921-553397

${ }^{b}$ Physical Chemistry II, Universität Bayreuth, Universitätsstrasse 30, 95440 Bayreuth, Germany

${ }^{c}$ Process Biotechnology, Universität Bayreuth, Universitätsstrasse 30, 95440 Bayreuth, Germany

$\dagger$ Electronic supplementary information (ESI) available: Materials, methods, synthesis, and supplementary characterization. See DOI: 10.1039/c5cc03901k
}

So far, many ongoing developments in dimethacrylate monomers could not adequately resolve the remaining issue of high volume shrinkage. ${ }^{12}$ Therefore, universally applied resins like bisphenol-A-glycidyl methacrylate (BisGMA) have so far played a pivotal role, as they provided a low volume shrinkage, ${ }^{13}$ rapid curing, and superior mechanical properties after curing. ${ }^{14}$ Nevertheless, the core of BisGMA resins is based on bisphenol-A (BPA), which has raised health concerns recently in the food packaging industry, consumer plastics, as well in dental materials about its widespread use, thus the exposure limits for BPA are reduced continuously. ${ }^{15}$

A promising resin system that sets a great promise on low volume shrinkage consists of vinylcyclopropane (VCP) derivatives, as the radical photo-polymerization results mainly in 1,5-ring-opened polymer units, whereby the volume shrinkage can significantly be reduced compared to methacrylate resins. ${ }^{16}$ However, so far not a single VCP resin could be successfully introduced to any real-world industrial application. Furthermore, due to the low curing kinetics and efficiency, VCPs have lost interest in academics as well. ${ }^{17}$

Herein, the first VCP-amide resin (VCPMe ${ }_{3}$ hexAmid) (Scheme 1) with very fast photo-radical polymerization kinetic, a high monomer conversion in less than 2 min in air, low volume shrinkage, low viscosity, an effective refractive index, and good mechanical properties is reported. We studied in detail the preparation of the

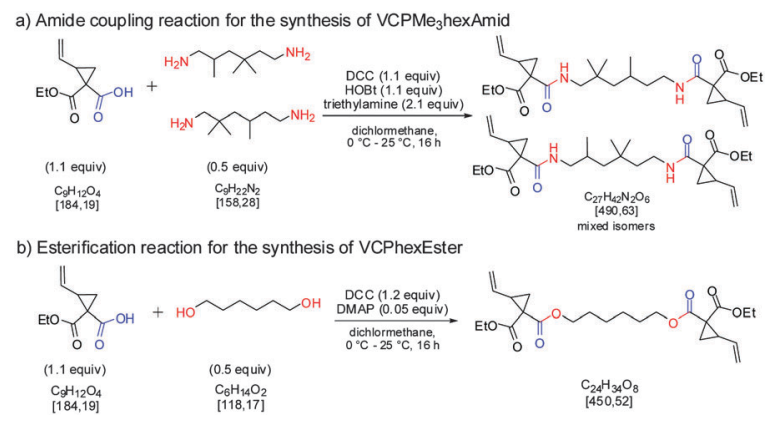

Scheme 1 Preparation of bi-functional VCPs. DCC $=N, N^{\prime}$-dicyclohexylcarbodiimide; $\mathrm{DMAP}=4$-dimethylaminopyridine; $\mathrm{HOBt}=$ benzotriazol-1-ol. 
VCP resin, its enhanced curing behaviour by photo-differential scanning calorimetry (photo-DSC) and dielectric analysis (DEA), its volume shrinkage, viscosity, and cytotoxicity. Furthermore, the change in the optical refractive index during curing by spectroscopic ellipsometry (SE), as well as the mechanical properties and the network structure of the cross-linked network by ${ }^{13} \mathrm{C}-\mathrm{CP} / \mathrm{MAS}$ (cross polarization/magic angle spinning) solid state NMR spectroscopy were studied to provide a detailed overview of the most relevant resin features.

In order to optimize the reactivity by hydrogen bonding and to prevent any disproportional increase in viscosity a sterically hindered and isomeric amide spacer was used (VCPMe ${ }_{3}$ hexAmid). A VCP-amide monomer, based on a linear C-6 spacer using simple hexamethylenediamine, provided slow crystallization and made itself unsuitable for any comparable detailed bulk polymerization study.

The $\mathrm{VCPMe}_{3}$ hexAmid was synthesized in high purity and yield according to Scheme 1, the structural characterization using NMR spectroscopy showed the formation of an isomeric mixture due to the stereo- and constitution specificity of the VCP- and spacer-molecules. A more complete description of the VCP resin is presented in the ESI $\dagger$ in Fig. S1-S7.

The $\mathrm{VCPMe}_{3}$ hexAmid was exposed to a commercial lightemitting diode (LED) source for studying the photo-curing kinetics and the absolute monomer conversion, which was determined by time-dependent extraction of the resulting material using deuterated chloroform. A mixture of camphorquinone (CQ) and ethyl 4-(dimethylamino)-benzoate (EDMAB) in a molar ratio of $1: 2$ was used for photo-polymerization at $35{ }^{\circ} \mathrm{C}$. The very fast curing kinetics of $\mathrm{VCPMe}_{3}$ hexAmid was evident from a conversion of $\sim 80 \%$ within 30 s and very high overall conversions of around $96 \%$ (Fig. 1a). The experiments were carried out both in inert gas as well as in air. The very fast curing-kinetics was retained even in air.

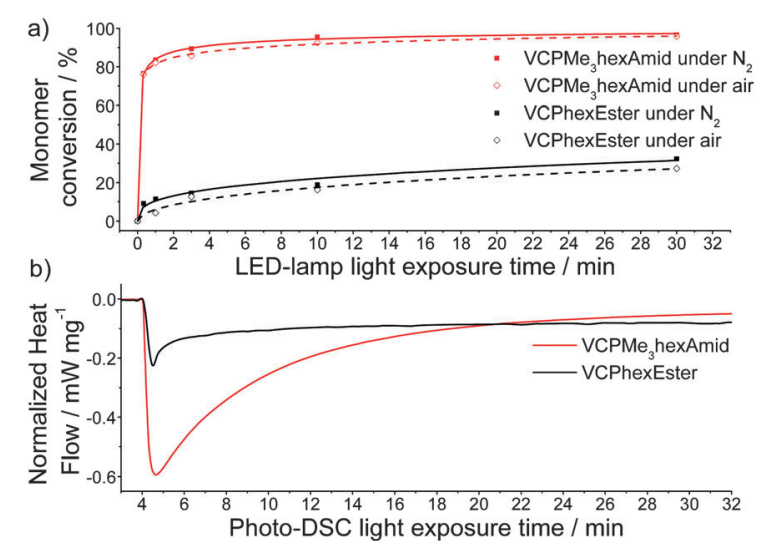

Fig. 1 Photo-polymerization of VCPMe 3 hexAmid and VCPhexEster (1 mol\% photo-initiator $C Q$ : EDMAB in a molar ratio of 1:2). (a) Time-dependent curing experiments under a controlled atmosphere of nitrogen and air using a commercial blue-light LED source $\left(2.013 \mathrm{~mW} \mathrm{~cm}^{-2}\right.$ for $\left.465 \mathrm{~nm}\right)$. The determination of residual monomer has been carried out by GC for VCPhexEster and by ${ }^{1} \mathrm{H}-\mathrm{NMR}$ for VCPMe 3 hexAmid. (b) Typical heat flows $\left(\mathrm{mW} \mathrm{mg}^{-1}\right)$ recorded during photo-DSC experiments $\left(6.93 \mathrm{~mW} \mathrm{~cm}{ }^{-2}\right.$ for the whole xenon spectrum).
These results were compared with that of the bi-functional VCP-ester derivative (VCPhexEster) (Scheme 1b, Fig. 1a). VCPhexEster yielded monomer conversions of $\sim 30 \%$ after $30 \mathrm{~min}$ of curing, while $\mathrm{VCPMe}_{3}$ hexAmid reached the same conversion in less than 20 s. Furthermore, VCPhexEster could not reach a high overall monomer conversion even after prolonged curing times up to several hours (the maximum conversion in nitrogen was $42 \%$ in $12 \mathrm{~h}$ ). Therefore VCPhexEster is several orders of magnitude less reactive than the $\mathrm{VCPMe}_{3}$ hexAmid. Furthermore, the polymerization heat flow during the curing process was monitored by carrying out photo-polymerization in a DSC-cell for both VCPhexEster and $\mathrm{VCPMe}_{3}$ hexAmid (Fig. 1b). Recording the relatively low heat flow changes during the curing of VCPhexEster also illustrated the general low reactivity of ester-based VCP resin.

The difference in reactivity of $\mathrm{VCPMe}_{3}$ hexAmid and VCPhexEster could be due to the molecular structure of $\mathrm{VCPMe}_{3}$ hexAmid allowing intermolecular hydrogen bonding via amide units, leading to a partial preorganization of monomer molecules (Fig. 2a). This could also lead to a profitable orientation of the singly occupied molecular orbital (SOMO) of the ring-opened VCP-structure to further monomer units or rather their $\pi$-orbitals, which enhances the curing process (Fig. 2b). The shift of the amide protons of $\mathrm{VCPMe}_{3}$ hexAmid to higher fields with increasing temperature in ${ }^{1} \mathrm{H}-\mathrm{NMR}$ experiments (see Fig. S8 and S9 in the ESI $\dagger$ ) and the meaningful difference in the monomer viscosities, $\mathrm{VCPMe}_{3}$ hexAmid was significantly more viscous (2.4 Pa s) than VCPhexEster (0.08 Pa s), verified the H-bonding. Furthermore, photo-DSC curing experiments, with an additional amount of potassium thiocyanate (KSCN) as a chaotropic agent, have been carried out to underline the hydrogen bonding effect (Fig. 2c). Thereby, chaotropic agents can disrupt the H-bonding forces. ${ }^{18}$ An unambiguous effect toward increasing KSCN amounts to the curing behavior was observed, yielding $19 \%$ lower heat flow values $\left(\Delta H_{\text {max }}\right)$ and $37 \%$ lower rates of polymerization $\left(R_{\mathrm{P}}\right)$, respectively, for $1 \mathrm{wt} \%$ of a KSCN solution (392 $\mathrm{mg} \mathrm{mL}^{-1}$ in DMSO) and $1 \mathrm{wt} \%$ pure DMSO $\left(R_{\mathrm{P}}\right.$ values were determined from the slope of the linear fit after reaching $H_{\max }$ ). The enhanced $R_{\mathrm{P}}$ for acrylate monomers capable of having $\mathrm{H}$-bonding is documented in the literature, ${ }^{19}$ however this effect appears to be not so pronounced as for VCPs.

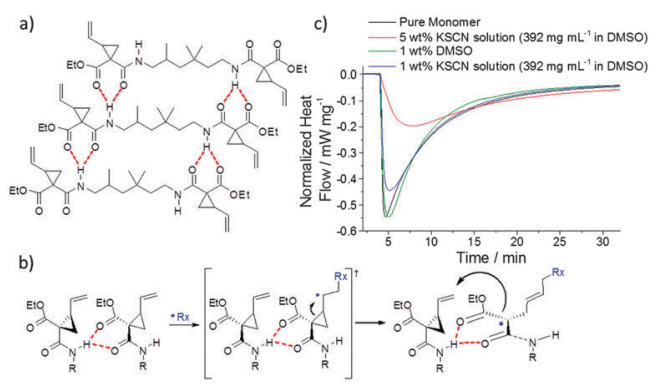

Fig. 2 Effect of hydrogen bonding on curing of VCPMe 3 hexAmid. (a) Proposed scheme showing partial preorganized monomer units. (b) Proposed mechanism for amide-based VCP polymerization in which the propagation rate is enhanced because of profitable orbital arrangements. (c) Photo-DSC measurements independent of KSCN as a chaotropic additive, proving the effect of hydrogen bonding for $\mathrm{VCPMe}$ hexAmid. 


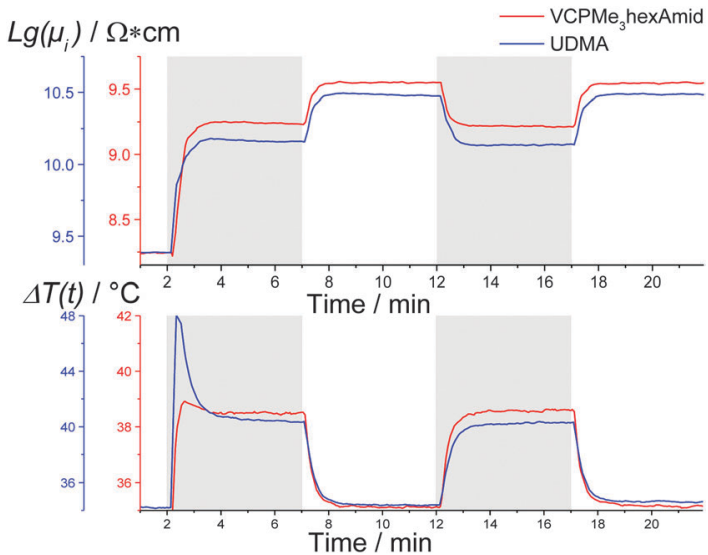

Fig. 3 Recorded changes in ion-viscosity $\left(\mu_{\mathrm{i}}\right)$ in DEA measurements monitored for a constant frequency of $1 \mathrm{~Hz}$ (upper graph) and polymerization temperature (lower graph) for the curing of VCPMe 3 hexAmid and UDMA ( 1 mol\% initiator CQ: EDMAB in a molar ratio of $1: 2$ ). The grey background indicates the light exposure.

Due to the fact that with time curing reactions are exothermic and could lead to undesirable local heating and an increase in temperature, additional experiments were carried out to monitor temperature changes during polymerization of $\mathrm{VCPMe}_{3}$ hexAmid by the DEA technique. Advantageously, the temperature increase $\Delta T$ during curing was very low (slightly above $4{ }^{\circ} \mathrm{C}$ ) for $\mathrm{VCPMe}_{3}$ hexAmid (Fig. 3). Furthermore, for $\mathrm{VCPMe}_{3}$ hexAmid the curing began immediately with an increase in the ion-viscosity $\left(\mu_{\mathrm{i}}\right)$ reaching the top of a plateau in about 1-2 min, whereas the continued exposure did not further affect $\mu_{\mathrm{i}}$. Thus, the curing was practically completed within the first $2 \mathrm{~min}$. The slight increase in $\mu_{\mathrm{i}}$ upon switching off the light source was due to a decrease in temperature from $39{ }^{\circ} \mathrm{C}$ (the maximum attained during curing) to around $35{ }^{\circ} \mathrm{C}$ (start temperature). No attempts were made to carry out similar experiments with VCPhexEster due to its very slow and incomplete polymerization as shown above. In contrast to $\mathrm{VCPMe}_{3}$ hexAmid, the well-known photo-cross-linkable resin urethane-dimethacrylate (UDMA) showed during curing a rise in temperature of $13{ }^{\circ} \mathrm{C}$ to about $48{ }^{\circ} \mathrm{C}$ under similar reaction conditions. Especially with regard to biological coatings and dental applications any reduction in an excessive exothermic temperature rise reduces the hazard potential significantly. ${ }^{20}$

The volume shrinkage as measured by Archimedes's method amounted to $4.9 \%$ for pure $\mathrm{VCPMe}_{3}$ hexAmid. The same measurement for VCPhexEster unfortunately could not be done due to incomplete conversions. Therefore, a comparison of our synthesized $\mathrm{VCPMe}_{3}$ hexAmid to widely used methacrylate resins, like UDMA, dodecanediol-dimethacrylate (1,12-DMA), and a mixture of BisGMA and triethylene-glycol-dimethacrylate (TEGDMA), was made.

$\mathrm{VCPMe}_{3}$ hexAmid showed a reduction by $45 \%$ in volume shrinkage to UDMA, 17\% to a 6:4 mixture of BisGMA: TEGDMA and similar to BisGMA. Furthermore, upon comparing the resin viscosity in dependence of volume shrinkage (Fig. 4a), a unique advantage of $\mathrm{VCPMe}_{3}$ hexAmid could be observed. Both BisGMA with $1200 \mathrm{~Pa} \mathrm{~s}^{21}$ and UDMA with $13.8 \mathrm{~Pa} \mathrm{~s}$ are too viscous to be used on their own. In contrast, $\mathrm{VCPMe}_{3}$ hexAmid exhibits a)

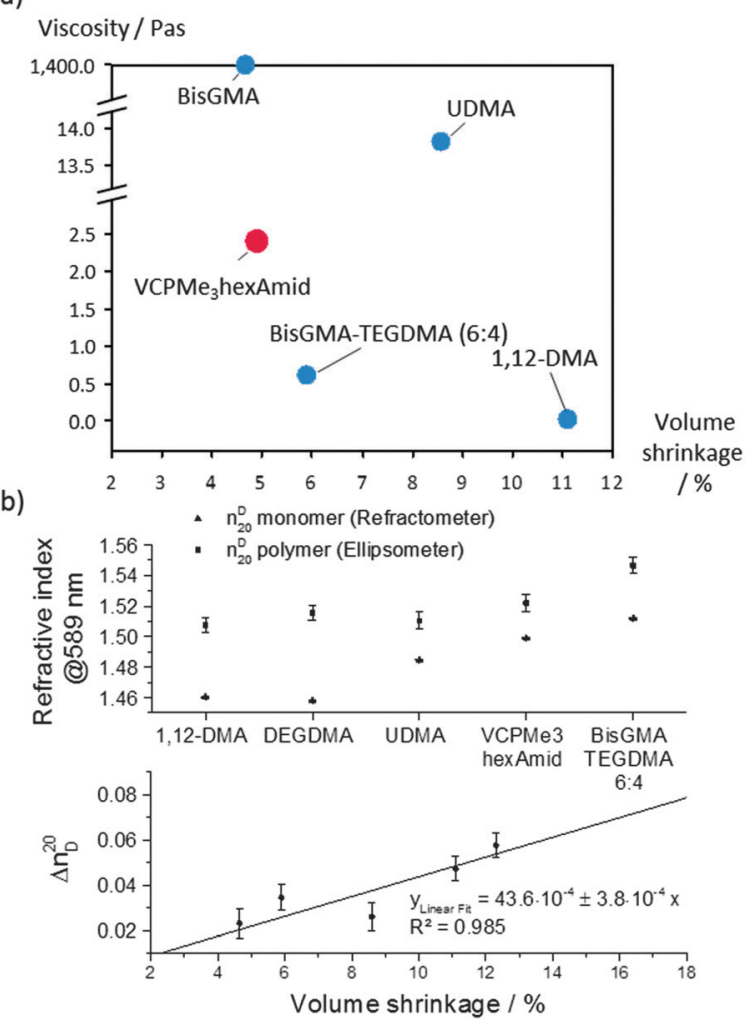

Fig. 4 Classification of resin properties in comparison to commercially available methacrylate resins. (a) Ashby plot of specific monomer viscosities as a function of the volume shrinkage. (b) Upper graph: refractive indices (@589 nm), respectively, for the monomer and polymer networks. Lower graph: graphical evaluation of the refractive index alteration $\Delta n_{\mathrm{D}}^{20}$ from the volume shrinkage of the respectively polymers. Please find in the $\mathrm{ESI} \dagger$ and Table $\mathrm{S} 1$ a summary of the properties of the materials shown here.

with 2.4 Pa s a clearly lower viscosity and simultaneously low volume shrinkage. The optical refractive index $n$ of the cured $\mathrm{VCPMe}_{3}$ hexAmid averaged to 1.522 as measured by SE and lies in the range of other existing systems, e.g. UDMA with 1.508 (Fig. 4b). The regression line for the changes in the refractive indices $\Delta n_{\mathrm{D}}^{20}$ in dependence of the volume shrinkage $\Delta V$ showed a simple and accurate correlation $\left(R^{2}=0.985\right)$ that the effect of the volume shrinkage mainly induced the refraction alteration.

Furthermore, VCPMe 3 hexAmid showed similar $\mathrm{LD}_{50}$ values $(0.067 \pm 0.005 \mathrm{mM})$ to those of UDMA $(0.071 \pm 0.009 \mathrm{mM})$ in case it has to be recommended for dental applications (see the $\mathrm{ESI} \dagger$ for cytotoxicity determination, Fig. S15 and S16).

As the polymer network structure could influence the overall properties, high-resolution solid state ${ }^{13} \mathrm{C}-\mathrm{CP} / \mathrm{MAS}$ NMR was used for studying the network structure by comparing the participation of double bonds in the cross-linking process. Almost all vinyl double bonds participated in the cross-linking reaction as evidenced by the presence of a very significant peak at $129.3 \mathrm{ppm}$ originating from the carbon signal of the $\mathrm{C}=\mathrm{C}$ double bond of the ring-opened VCP-unit. Only a very small shoulder at $119.7 \mathrm{ppm}$ (dedicated to unreacted vinyl double bonds) was identified, implying an extraordinary high cross-linking density of the cured $\mathrm{VCPMe}_{3}$ hexAmid. A closer examination of the network structure is presented in 
the ESI $\dagger$ and Scheme S4 and Fig. S10; with respect to the polymerization mechanism of VCPs the formation of cyclobutane structures besides the 1,5-ring-opened units should also be taken into consideration, but unfortunately it could not be analyzed using present techniques. ${ }^{16}$

Cured $\mathrm{VCPMe}_{3}$ hexAmid specimens showed E-moduli of $130.9 \pm 0.7 \mathrm{MPa}$ which increased to $181.9 \pm 0.5 \mathrm{MPa}$ after additional storage at $100{ }^{\circ} \mathrm{C}$ (the complete evaluation is provided in the ESI $\dagger$ and Fig. S12) and Vickers hardness (HV) values of $1.52 \pm 0.02 \mathrm{HV}$. These values are in the range of commercially available and cured UDMA.

In summary, after $\sim 10$ years of silence in academics and patent registrations the first highly reactive and low volume shrinking VCP resin was obtained, which can serve as a model monomer for further resin systems. The introduction of H-bond forming amide groups into the monomer structure induced partial preorganization, leading to high propagation efficiency during curing. Any disproportionate increase in resin viscosity could be prevented by using a sterically hindered, isomeric spacer element. The resulting $\mathrm{VCPMe}_{3}$ hexAmid represents for the first time a highly efficient and fast curing VCP resin. Moreover, the most valuable properties conversion, mechanical strength, viscosity, and optical refractive index were all carefully balanced in optimum ranges. Since radical polymerizable, low-shrinking resins remain a challenge for several applications, this novel resin could be of immense interest, offering a real and sustainable alternative to the currently used dimethacrylate resins. So far, the outstanding possibilities offered by VCP resins have not yet been exploited. Therefore a broad application range for both industry and academia may be imagined.

This work was supported by the German Federal Ministry for Economic Affairs and Energy (BMWi) (www.zim-bmwi.de). We thank Dr A. Bublewitz, Dr A. Theis and the Kettenbach GmbH for providing further resources and support. The authors are indebted to Prof. P. Strohriegel and Dr R. Giesa for providing access to photo-DSC and DMTA, to Prof. J. Senker and Dr R. Siegel for solid-state NMR measurements, and further to NETZSCH company and Dr S. Schmölzer for DEA measurements. We also thank
C. Goldhahn and P. Schmode for their experimental support as research students.

\section{Notes and references}

1 (a) Y. Jian, Y. He, L. Zhao, A. Kowalczyk, W. Yang and J. Nie, Adv. Polym. Technol., 2013, 32, 21331; (b) E. Andrzejewska, Prog. Polym. Sci., 2001, 26, 605.

2 (a) J. G. Kloosterboer, Adv. Polym. Sci., Springer, Berlin Heidelberg, 1988, vol. 84, pp. 1-61; (b) Y. Fuchs, O. Soppera and K. Haupt, Anal. Chim. Acta, 2012, 717, 7.

3 C. Decker, Macromol. Rapid Commun., 2002, 23, 1067.

4 J. Guo, M. R. Gleeson and J. T. Sheridan, Phys. Res. Int., 2012, 2012, 1. 5 J. Lange, S. Toll, J.-A. E. Månson and A. Hult, Polymer, 1997, 38, 809.

6 (a) N. Moszner and T. Hirt, J. Polym. Sci., Part A: Polym. Chem., 2012, 50, 4369; (b) K. L. van Landuyt, J. Snauwaert, J. de Munck, M. Peumans, Y. Yoshida, A. Poitevin, E. Coutinho, K. Suzuki, P. Lambrechts and B. van Meerbeek, Biomaterials, 2007, 28, 3757.

7 K. S. Anseth, C. N. Bowman and N. A. Peppas, J. Polym. Sci., Part A: Polym. Chem., 1994, 32, 139.

8 (a) A. Lendlein, H. Jiang, O. Jünger and R. Langer, Nature, 2005, 434, 879; (b) C. Fo Tsang, Microelectron. Int., 2000, 17, 27.

9 (a) C. Decker, Prog. Polym. Sci., 1996, 21, 593; (b) K. L. van Landuyt, J. Snauwaert, J. de Munck, M. Peumans, Y. Yoshida, A. Poitevin, E. Coutinho, K. Suzuki, P. Lambrechts and B. van Meerbeek, Biomaterials, 2007, 28, 3757.

10 F. Rueggeberg and K. Tamareselvy, Dent. Mater., 1995, 11, 265.

11 B. Lu, P. Xiao, M. Sun and J. Nie, J. Appl. Polym. Sci., 2007, 104, 1126.

12 J. W. Stansbury, M. Trujillo-Lemon, H. Lu, X. Ding, Y. Lin and J. Ge, Dent. Mater., 2005, 21, 56.

13 R. L. Bowen, US 3066112, 1959.

14 (a) R. L. Bowen, J. Am. Dent. Assoc., JADA, 1963, 66, 57; (b) A. Peutzfeldt, Eur. J. Oral Sci., 1997, 105, 97.

15 (a) C. Liao and K. Kannan, Environ. Sci. Technol., 2011, 45, 9372; (b) L. Chen and B. Suh, JSM Dent, 2013, 1, 1004; (c) C. Bolognesi, L. Castle, J.-P. Cravedi, K.-H. Engel, P. Fowler, R. Franz, K. Grob, R. Gürtler, T. Husøy, W. Mennes, M. R. Milana, A. Penninks, F. Roland, V. Silano, A. Smith, M. de Fátima Tavares Poças, C. Tlustos, F. Toldrá, D. Wölfle and H. Zorn, EFSA J., 2015, 13, 3978.

16 P. Pineda Contreras, P. Tyagi and S. Agarwal, Polym. Chem., 2015, 6, 2297.

17 N. Moszner, Macromol. Symp., 2004, 217, 63.

18 F. Liu, J. Seuring and S. Agarwal, J. Polym. Sci., Part A: Polym. Chem., $2012,50,4920$.

19 (a) J. F. G. A. Jansen, A. A. Dias, M. Dorschu and B. Coussens, Macromolecules, 2003, 36, 3861; (b) S. H. Dickens, J. W. Stansbury, K. M. Choi and C. J. E. Floyd, Macromolecules, 2003, 36, 6043; (c) I. Sideridou, V. Tserki and G. Papanastasiou, Biomaterials, 2002, 23, 1819.

20 (a) A. C. Shortall and E. Harrington, J. Oral Rehabil., 1998, 25, 908-913; (b) Z. Czech and R. Milker, J. Appl. Polym. Sci., 2003, 87, 182. 21 S. G. Pereira, T. G. Nunes and S. Kalachandra, Biomaterials, 2002, 23, 3799. 